\title{
Adipose tissue engineering and adipogenesis - a review
}

\section{Andreea D. Lazar, Sorina Dinescu ${ }^{\bowtie}$, Marieta Costache}

Department of Biochemistry and Molecular Biology, Faculty of Biology, University of Bucharest, Spl. Independentei 91-95, 050095, Bucharest, Romania

${ }^{\circledR}$ Correspondence to: Sorina Dinescu, Phone: 0213181575 Fax: 0213181575, int.102 E-mail: sorina.dinescu@ bio.unibuc.ro

Received: 4 December 2017 / Revised: 4 March 2018 / Accepted: 5 April 2018 / Available online: 22 April 2018

\begin{abstract}
Adipose tissue engineering represents a promising solution for the regeneration of adipose tissue. The goal is to achieve a substitute for this specific tissue, appropriate for implantation at the lesion site, by combining cells, biomaterials and induction factors that promote adipogenesis. The cells undergo adipogenic differentiation, specific genes are up-regulated by transcription factors and certain signaling pathways are activated or silenced. The aim of this review was to provide the readers with an overview of the main molecular events associated with adipogenesis, stressing on the most important aspects of signaling during this process, as well as with the main aspects of adipose tissue engineering up to date.
\end{abstract}

Keywords: adipose tissue engineering, adipogenesis, transcription factors, adipose tissue signaling, regeneration

\section{Introduction}

Organ and tissue transplants have the potential to save millions of lives worldwide, but unfortunately clinicians find themselves in a situation where these transplants are impossible to perform due to the lack of natural resources. Therefore, a new field called regenerative medicine has emerged in order to repair or replace damaged tissues and organs and improve their functionality. Tissue engineering is just one of the many fields that are encompassed by the regenerative medicine domain.

Tissue engineering (TE) strategies focus on the use of cells, biomaterials and induction factors. In the past decades numerous biomaterials - based on natural or synthetic compounds - have been designed and developed to serve as scaffolds, structures capable of mechanically supporting the cells seeded on their surface and facilitating cell proliferation and differentiation in the presence of induction factors. These cells can be isolated from many sources, but the ones that are used frequently are adult stem cells or cell lines (cells already committed to a specific fate, for example: preadipocytes, premyocytes, prechondrocytes, etc.). Embryonic stem cells, though exhibit totipotent status, have restricted use because of ethical considerations.

There are two possible strategies for adipose tissue engineering (ATE), depending on the quantity of tissue needed for regeneration, and these are in vivo/in situ adipogenesis or $3 \mathrm{D}$ in vitro TE. The structure obtained using ATE strategies must have certain characteristics such as biocompatibility, sustainability and bioactivity.
In order for the tissue to be formed, the cells embedded in the scaffold undergo the process of adipogenesis and become mature adipocytes capable of accumulating lipids and secreting specific proteins. Therefore, it is of upmost importance to know and understand the key events during adipogenesis.

\section{Adipose tissue engineering (ATE)}

The purpose of this multidisciplinary field is the development of biological substitutes for adipose tissue (Casadei et al. 2012). The regeneration is necessary for patients who have lost this specific tissue due to traumas, for example severe burns or various diseases such as breast cancer, congenital malformations, etc. (Patrick 2001; Rustad et al. 2010). The lasting effects of this loss affect the patients not only aesthetically, but also emotionally, and it can damage the functionality of the tissue as well (Katz et al. 1999; Patrick 2001).

Adipose tissue engineering (ATE) combines cells, biomaterials and induction factors in order to obtain a structure fit for the reconstruction of the damaged or missing adipose tissue (Fig. 1).

\section{Short history}

The first adipose tissue transplant was performed in 1893 by G.A. Neuber, a German surgeon, who used fat grafts to correct facial deformities (Locke and de Chalain 2008). Although much has improved from that time until now, the use of autologous fat grafts, alloplastic materials or allogenic transplants in plastic and reconstructive sur- 
gery still has numerous limitations (Monfort and Izeta 2012). Transplanting autologous adipose tissue from one part of the body to a damaged area is only a temporary solution because the fat is gradually reabsorbed in time (Coleman 1995). This reabsorption of the implanted tissue seems to occur mainly because of the lack of vascularization (Nguyen et al. 1990). Adipose tissue is highly vascularized and innervated so the simple transfer of fat from one area to another is not sufficient for the reconstruction of the affected anatomical region. Only minor flaws can be corrected by repeatedly injecting autologous fat to maintain the volume (Huss and Kratz 2002). Aside from the reabsorption of the graft, deformities or necrosis of the donor tissue may appear (Katz et al. 1999). Alloplastic materials and allogenic transplants can cause allergic reactions, the transplanted tissue or material may be rejected by the patient's organism, the implant may migrate or be incapable of integrating into the tissue (Patrick 2001).
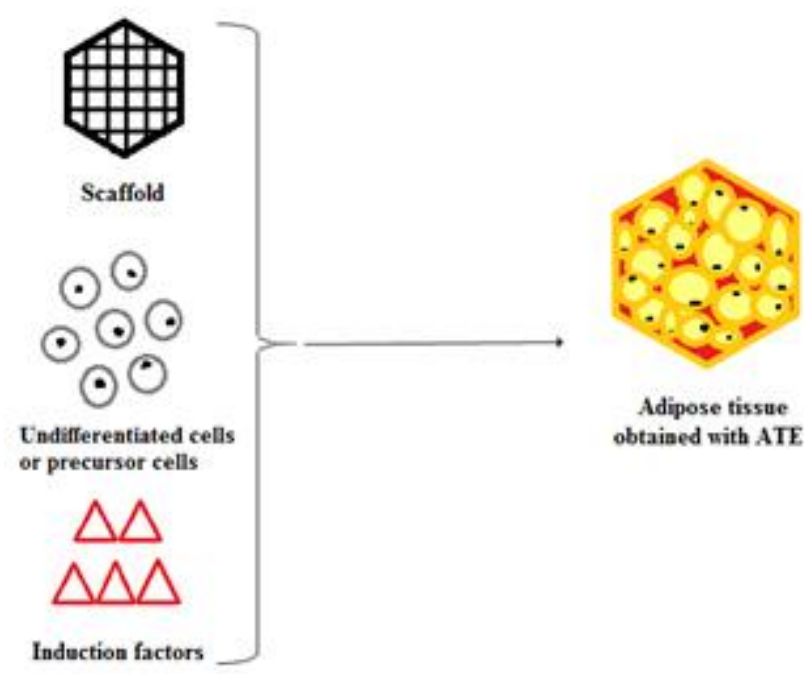

Fig. 1. The principle of adipose tissue engineering (ATE). Appropriate cells (undifferentiated or precursor cells) are implanted in the scaffold in the presence of induction factors (that facilitate the differentiation of cells), in order to obtain a substitute for adipose tissue.

To overcome these limitations and adequately restore the damaged or missing tissue, ATE represents an innovative and promising solution. The main strategy of ATE is based on populating a biomaterial (scaffold), which acts as a substitute for the extracellular matrix (ECM) with cells that have the ability to differentiate into adipocytes (Flynn and Woodhouse 2008). The cells are isolated from various sources, cultured in the laboratory, then implanted in the scaffold, a structure that offers temporary support and is capable of supporting the growth and development of the cells. The scaffold resembles the ECM, it promotes cellular migration and attachment and facilitates cell-cell and cell-matrix interactions (Gomillion and Burg 2006). The cellsscaffold complex, also named bioconstruct, can then be implanted at the lesion site and used for the regeneration of adipose tissue. Modulating the cellular proliferation and differentiation is possible by using induction factors. Once implanted, the scaffold gradually degrades as the tissue is formed and a new ECM is secreted by the cells (Fuchs et al. 2001; Choi et al. 2010). Therefore, it can be concluded that the biomaterial determines the shape and the size of the regenerated tissue (Patrick 2001).

The first in vitro adipose bioconstruct was developed by Patrick et al. in 1999 using rat preadipocytes and a scaffold based on poly (lactic-co-glycolic) acid (PLGA). Unfortunately this bioconstruct did not last (Patrick et al. 2002). Since then, scientists have undergone different experiments in order to design and develop an adequate substitute capable of repairing and regenerating the damaged adipose tissue. Nonetheless, the results were not considered safe and efficient for clinical application (Sommer and Sattler 2000; Choi et al. 2010).

\section{Qualities of an adequate ATE product}

The following criteria needs to be met in order to generate an ATE product that can be used (Choi et al. 2010):

\section{Compatibility}

The bioconstruct must not determine an immune response translated as the rejection of the implant by the patient's organism, therefore it must be compatible (Levenberg and Langer 2004). The construction of an adipose substitute has to be reported to the affected region of the patient because this area influences the level of regeneration of the adipose tissue and the metabolic function of the regenerated tissue (Kirkland et al. 1994; Schipper et al. 2008). The degradation method of the bioconstruct once implanted must also be compatible with the host, which means that the rate at which the biomaterial degrades should be the same as the rate at which the tissue regenerates (Lavik and Langer 2004).

\section{Bioactivity}

The regenerated tissue must be bioactive, which means it must be structurally and functionally identical with the native adipose tissue. Thus, it is necessary to incorporate a functional vascular system made out of blood vessels, lymphatic vessels and nerves (Hausman et al. 2001). This vascular system can be obtained differently, depending on the desired application: in vitro by adding endothelial cells or in vivo by recruiting the cells of the host organism. To evaluate the level of vascularization, histological analyses for specific endothelial markers can be performed (Lin et al. 2008; Marra et al. 2008).

\section{Sustainability}

The new tissue has to be viable and functional for a long period of time (Choi et al. 2010). The functionality of the tissue obtained using ATE strategies can be evaluated by a variety of methods. Intracellular lipids from mature adipocytes can be visualized by staining in red using Oil 
Red $\mathrm{O}$ assay and they can be quantified by enzymatic tests (Kang et al. 2008). The detection of specific transcription factors - such as peroxisome proliferatoractivated receptor (PPAR $\gamma)$, glucose transporter 4 (GLUT4), fatty-acid binding protein (FABP4), acyl-CoA synthetase (ACS) - is possible by using Real Time PCR, thus monitoring the process of adipogenic differentiation and lipogenic activity (Kang et al. 2008; Shanti et al. 2008). Adipose tissue is an endocrine organ as well, therefore the ability of the regenerated tissue to secrete adipokines (leptin, adiponectin, etc.) has been investigated (Fischbach et al. 2004; Vermette et al. 2007).

\section{Adipose tissue engineering strategies}

The techniques and methods used to regenerate adipose tissue are what scientists call strategies. In order to be able to generate a bioactive adipose substitute, fit from a structural, mechanical and functional point of view, the knowledge of the morphology, but especially the physiology of adipose tissue in vivo, is crucial. There are two major types of adipose tissue: white adipose tissue (WAT) and brown adipose tissue (BAT) (Choi et al. 2010).

White adipose tissue is an endocrine organ involved in various metabolic functions and pathological conditions, but its main purpose is to store triacylglycerols (TAGs), thus it is considered an energy deposit (Tilg and Moschen 2006). It is found in many regions such as subcutaneous (arms, abdomen, gluteal), intraabdominal (retroperitoneal, omental, visceral), retro-orbital, pericardial, intramuscular, periarticular regions and bone marrow (Gesta et al. 2007). It is mainly formed out of mature adipocytes, but it also contains preadipocytes, endothelial cells, interstitial cells, pericytes, blood vessels, lymphatic vessels and nerves (Hausman et al. 2001). All of these are supported by an ECM made out of different types of collagen (I, II, IV, V, VI) and other proteins (Flynn et al. 2006).

On the other hand, BAT doesn't store TAGs but uses them for thermogenesis. The largest quantity of BAT is found at birth, spread over the body of the new-born, but as the organism undergoes growth and development, the quantity of BAT diminishes while WAT increases (Cannon and Nedergaard 2004; Farmer 2008).

Although there is an increasing interest in the presence and purpose of BAT in the metabolism and function of adipose tissue, ATE strategies are centered on the regeneration of WAT because this type of adipose tissue is predominant in the mature human organism (Choi et al. 2010).

\section{A. In vivo or in situ adipogenesis - for the regeneration of a small amount of tissue}

There are two strategies that can be used in order to favor the generation of adipose tissue, either by injecting differ- entiation inducers at the implantation site or by inserting a cell-scaffold bioconstruct to support tissue architecture.

\section{a. Injection of induction factors}

In vivo adipogenesis can be induced by stimulating the differentiation of preadipocytes or stem cells already present in the native tissue. This stimulation of the cells is achieved by injecting active biomolecules called induction factors, which influence the proliferation and differentiation of the progenitor cells, into the anatomical region in need of de novo adipose tissue (Katz et al. 1999). One example for this approach is the experiment conducted by Tabata and his colleagues in which they generated adipose tissue by injecting a mixture of Matrigel (a commercially available gelatinous ECM secreted by mouse sarcoma cells used as a basement membrane) and biodegradable soft microspheres filled with basic fibroblast growth factor (bFGF, that promotes the proliferation and differentiation of cells) into subcutaneous tissue of mice (Tabata et al. 2000).

\section{b. Injection of cells-scaffold complex}

Cells that have the ability to proliferate and differentiate into adipose tissue (stem cells or preadipocytes) are isolated from the patient's tissue, multiplied and then implanted on a scaffold with or without induction factors (Katz et al. 1999).The resulting bioconstruct is then implanted in vivo in the injured area. The first experiment that used this strategy was performed in 1999, when scientists managed to implant a complex made out of autologous preadipocytes and a PLGA based scaffold in mice (Patrick et al. 1999).

\section{B. 3D in vitro tissue engineering - for the regeneration of a big amount of adipose}

\section{tissue}

Three dimensional in vitro TE resembles the strategy mentioned above but in this case, induction factors are used for modulating the adipogenic differentiation of cells embedded in the 3D scaffold. The resulting adipose tissue is obtained in vitro and afterwards transplanted to the affected anatomical region (Griffith and Naughton 2002; Monfort and Izeta 2012).

For the induction of adipogenic differentiation of cells, a cocktail made out of insulin, dexamethasone and isobutyl-methyl-xanthine (IBMX) in various proportions is usually used (Scott et al. 2011). Insulin plays a role in the metabolism of carbohydrates and in the synthesis of TAGs. Dexamethasone is a glucocorticoid and has an anti-inflammatory effect. IBMX, a phosphodiesterase inhibitor, determines the accumulation of intracellular cAMP and, in combination with dexamethasone, it modulates the activity of PPAR $\gamma$, an extremely important transcription factor involved in adipogenesis, thus promoting the process of adipogenic differentiation (Gomillion and Burg 2006). 
Other frequently used biomolecules are agonists of $\operatorname{PPAR} \gamma$, such as thiazolidinedione and indomethacin, added in various concentrations from 1 to $200 \mu \mathrm{M}$ (Scott et al. 2011).

\section{Adipogenesis}

The adipogenic differentiation program is an intricate process, by which undifferentiated cells convert into mature adipocytes over multiple stages. Signaling pathways and numerous transcription factors are involved in the regulation of gene expression responsible for the specific phenotype (Moreno-Navarrete and FernándezReal 2012; Moseti et al. 2016). Changes occur in the cytoskeletal and ECM composition, shape, affinity for insulin and secretory capacity of the cells (Lefterova and Lazar 2009).

Adipogenesis is one of most studied processes of cellular differentiation due to the availability of a large number of in vitro models capable of precisely reproducing the essential stages of in vivo adipogenesis. Understanding these steps and the molecular mechanism underlying adipogenesis can bring benefits to researchers and doctors involved in the field of regenerative medicine and tissue engineering, as well as to the research related to the dysregulation of adipose tissue metabolism. Metabolic syndrome, obesity and diabetes are associated metabolic diseases. Therefore, the long term perspectives of adipogenesis studies can also be included in the global research efforts to reduce the rate of obesity and diabetes in population (Dinescu 2015).

\section{Stages}

Commitment, determination and differentiation of undifferentiated cells to mature adipocytes is specifically regulated and can be summed up in the following stages: growth arrest, mitotic clonal expansion, early differentiation and late differentiation (Fig. 2) (Zuk et al. 2001; Franchini 2003; Dicker et al. 2005).

\section{a. Growth arrest}

This step is necessary for the committed preadipocytes to withdraw from the cellular cycle before differentiation. Growth arrest is possible through contact inhibition. Still, contact inhibition is not essential for adipogenesis; it has been observed that preadipocytes cultivated at low density in medium without serum, differentiate in the absence of cell-cell contact, which suggests that growth arrest and absence of confluence are necessary conditions for the initiation of adipogenesis.

Two major transcription factors can act cooperatively in growth arrest PPAR $\gamma$ (Altiok et al. 1997) and CCAAT/enhancer binding protein $(\mathrm{C} / \mathrm{EBP} \alpha)$ (Umek et al. 1991). Although the expression of $\mathrm{C} / \mathrm{EBP} \alpha$ and $\mathrm{PPAR} \gamma$ strongly increases as adipogenesis progresses, the low level of expression of these transcription factors in adipocytes can be sufficient to mediate growth arrest, which precedes differentiation.

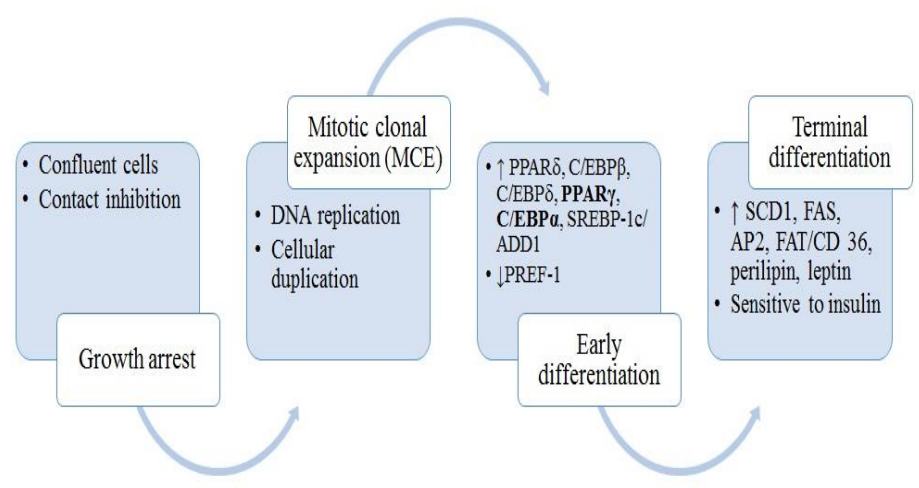

Fig. 2. Adipogenesis - stages and molecules involved. There are four stages: growth arrest, mitotic clonal expansion (MCE), early differentiation and terminal differentiation. After proliferation the cells reach a state of confluence and their growth is arrested through contact inhibition. With appropriate signals, the commited cells become determined and they undergo MCE, resulting in cellular duplication. Afterwards, the cells proceed to early and terminal differentiation, each with its own specific markers: peroxisome proliferator-activated receptor (PPAR), CCAAT/enhancer binding protein (C/EBP), sterol regulatory element binding protein-1c/adipocyte determination and differentiation factor 1 (SREBP-1c/ADD1), preadipocyte factor-1 (PREF-1), stearoyl CoA desaturase 1 (SCD1), fatty acid synthase (FAS), adipose-specific fatty acid binding protein (AP2), fatty acid transporter (FAT/CD36), perilipin, leptin.

\section{b. Mitotic clonal expansion (MCE)}

After growth arrest at confluence, the committed preadipocytes must receive an appropriate combination of mitogenic and adipogenic signals in order to become determined to proceed to the next stage, namely differentiation. Research done on cell lines have shown that preadipocytes undergo at least one round of DNA replication and cellular duplication which leads to the clonal expansion of the committed cells (Pairault and Green 1979). In vitro these mitogenic and adipogenic signals are represented by insulin, dexamethasone and IBMX (Howard et al. 2008).

During adipogenesis, the specific phenotype is attained through chronological changes in the expression of numerous genes. These modifications are reflected by the appearance of early, intermediate and late markers and in the accumulation of TAGs; they mainly occur at a transcriptional level, although in the case of some specific adipogenic genes, the regulation occurs also at a post-transcriptional level. Genes that are unnecessary for the function of the adipocytes are repressed (Wilkison et al. 1990; Moustaid and Sul 1991).

\section{c. Early changes in gene expression}

Growth arrest and clonal expansion are accompanied by complex modifications in the pattern of gene expression. The expression of primary transcript of lipoprotein lipase 
(LPL) is considered an early sign of adipogenic differentiation (MacDougald and Lane 1995; Ailhaud 1996). LPL is secreted by mature adipocytes and plays a central role in controlling lipid accumulation. Still, LPL is expressed spontaneously at confluence and is not influenced by the nature of the compounds implicated in adipogenesis, therefore it can reflect the stage of growth arrest rather than early differentiation (Amri et al. 1995). At least two families of transcription factors, C/EBP and PPAR, are induced early on in adipogenesis. PPAR $\gamma$ is specific for adipocytes and is expressed at a low but detectable level in preadipocytes. The expression of PPAR- $\gamma$ rapidly increases after hormonal induction of adipogenic differentiation. The induction of PPAR- $\delta$ seems to precede that of PPAR- $\gamma$ but the expression of PPAR $-\delta$ is more dispersed. Another transcription factor that is induced early on during differentiation is Sterol Regulatory Element Binding Protein-1c/ Adipocyte Determination and Differentiation Factor 1 (SREBP-1c/ ADD1), a bHLH-leucine zipper protein implicated in cholesterol metabolism and gene expression in adipogenesis (Dinescu 2015).

During adipogenic differentiation, the cells no longer retain the fibroblastic shape, but they become spherical. This shows that profound changes take place in the morphology of the cell, in the cytoskeletal components and ECM. The decrease in the expression of actin and tubulin is an early sign of adipogenesis, which precedes the morphological modifications and the expression of specific genes for adipocytes. Another early event is represented by the changes in expression of the genes that encode for collagen; type I and II decrease by $80-90 \%$ during 3T3-L1 cells differentiation, while type IV and nidogen expression increases (Dinescu 2015).

Preadipocyte Factor-1 (PREF-1), a protein that contains epidermal growth factor (EGF) repetitive motifs, is thought to have an implication in maintaining the preadipose phenotype (Smas et al. 1997). A dramatic decrease in the expression of PREF-1 is registered once adipogenic differentiation occurs, PREF-1 being abundant in preadipocytes and undetectable in mature adipocytes.

\section{d. Terminal differentiation}

During terminal differentiation, for adipocytes in culture there is a significant increase in lipogenesis and in the activity and protein levels of enzymes involved in TAGs metabolism: ATP citrate lyase, malic enzyme, acetyl CoA carboxylase, stearoyl CoA desaturase (SCD1), glycerol 3-phosphate acyl transferase, glycerol 3phosphate dehydrogenase, fatty acid synthase (FAS) and glyceraldehyde 3-phosphate dehydrogenase. The cells also become sensitive to insulin (Spiegelman et al. 1983). Furthermore, adipocytes synthesize specific proteins for adipose tissue such as adipose-specific fatty acid binding protein (AP2) (Spiegelman et al. 1993), FAT/CD36, a fatty acid transporter (Ibrahimi et al. 1996) and perilipin, which coats the lipid droplets of adipocytes (Greenberg et al. 1993). The level of leptin also increases during terminal differentiation, with a much higher level in vivo than in vitro (MacDougald et al. 1995).

The progressive dedifferentiation of primary mature adipocytes, followed by cellular division, has been reported (Sugihara et al. 1986). Treatment of mature adipocytes with TNF- $\alpha$ results in low expression of adipocyte markers, loss of lipids and morphological modifications, such as cytoplasmic fusiform extensions (Torti et al. 1989), close to preadipocytes. If the reversion to a preadipose phenotype takes place in vivo then it should play a role in regulating the number of adipocytes and the mass of adipose tissue.

\section{Transcriptional control of adipogenesis}

During adipogenesis, the cell receives a variety of signals, which lead to its commitment to the process of differentiation. Growth factors and hormones play a role in adipogenic differentiation by interacting with specific cell receptors and initiating a cascade of intracellular events. Identifying these molecules, which positively or negatively modulate the process, is important because it gives us information regarding the signaling pathways involved in adipogenesis (Dinescu 2015).

Upon reaching the nucleus, these signals lead to the coordinated regulation of gene expression specific for adipocytes. Although the complete set of genes involved in adipogenesis is still unclear, it is known that PPAR and $\mathrm{C} / \mathrm{EBP}$ families of transcription factors must act in a cooperative manner in order to transactivate adipocyte genes and promote adipogenesis (Mandrup and Lane 1997).

\section{PPAR family of transcription factors}

PPAR proteins belong to the family of hormone nuclear receptors type II that form heterodimers with retinoid $\mathrm{X}$ receptor (RXR) (Schoonjans et al. 1996). These proteins are activated by a variety of different compounds including anti-diabetic medication. PPAR-RXR complexes regulate transcription upon binding to DNA at specific sites called response elements, which recognize the nuclear receptor (Tontonoz et al. 1994).

Out of all the members of the family, PPAR $\gamma$ is the most specific for adipose tissue. Its expression is induced before the transcriptional activation of most adipocyte genes and this expression is sufficient for the cells to undergo growth arrest and initiation of adipogenesis (Tontonoz et al. 1995; Altiok et al. 1997).

Because of alternative splicing at the 5' end of the AP2 transcript, PPAR $\gamma$ has two isoforms - PPAR $\gamma 1$ and PPAR $\gamma 2$. The only difference between them is the additional 30 amino acids located at the N-terminal end of PPAR $\gamma 2$ (Fajas et al 1997). Expression of PPAR $\gamma 1$ can be found in the majority of tissues, though at a low level, while PPAR $\gamma 2$ is specific for adipose tissue and highly expressed (Braissant et al 1996). 
PPAR $\gamma$ plays an essential role in the transcriptional activation of the majority of genes that encode for adipogenic markers. For example, the activation of the enhancer specific for $A P 2$ depends on the binding of PPAR $\gamma$ (Dinescu 2015).

\section{C/EBP family of transcription factors}

The C/EBP family of transcription factors is made out of five members: C/EBP- $\alpha, \mathrm{C} / \mathrm{EBP}-\beta, \mathrm{C} / \mathrm{EBP}-\delta, \mathrm{CHOP}$ and $\mathrm{C} / \mathrm{EBP}-\gamma$. They all have a transcriptional activation domain and a leucine zipper motif that ensures the ability for homo/heterodimerization (Ramji and Foka 2002).

For adipogenesis the most important one is $\mathrm{C} / \mathrm{EBP} \alpha$, which functions in a cooperative manner with PPAR $\gamma$ to activate the majority of adipocyte specific genes (AP2, SCD1, GLUT4, etc.). This regulation is made possible by the existence of regulatory binding elements (response elements) found at specific DNA sites (Christy et al. 1991). Mutations at these sites lead to inactivation of the genes (Hwang et al. 1996). In the absence of hormonal induction, $\mathrm{C} / \mathrm{EBP} \alpha$ expression is sufficient to initiate differentiation in 3T3-L1 cells, thus demonstrating its crucial role in adipogenesis. Also, the antimitotic activity of this transcription factor facilitates the transition of the cells from MCE phase to the next stages of the process (Lin and Lane 1994).

Aside from $\mathrm{C} / \mathrm{EBP} \alpha$, two other members of the family are involved in adipogenesis: $\mathrm{C} / \mathrm{EBP} \beta$ and $\mathrm{C} / \mathrm{EBP} \delta$. They are expressed during early differentiation and they promote the expression of $\mathrm{C} / \mathrm{EBP} \alpha$ and PPAR $\gamma$ in the terminal stage (Zuo et al. 2006).

\section{Transcription factors that inhibit adipogenesis}

PPAR and C/EBP are considered to be the most important transcription factors that stimulate adipogenesis, but they are by no means the only ones involved in the molecular control of this process. Other transcription factors act as inhibitors for adipogenic differentiation and some examples are: (i) Globin transcription factor 2 (GATA2) and Globin transcription factor 3 (GATA3), repress the activity of PPAR $\gamma 2$ promoter (Chargé and Rudnicki 2004) and form protein complexes with $\mathrm{C} / \mathrm{EBP} \alpha$ and $\mathrm{C} / \mathrm{EBP} \beta$ (Brand-Saberi 2005). They also activate the Wnt signaling cascade, stopping the transition from preadipocytes to adipocytes (Otto and Rao 2004; Tajbakhsh 2005); (ii) Krüppel-like Factor 2 (KLF2), maintains the preadipocyte state if not otherwise repressed (Lian et al. 2004). KLF2 also prevents the activation of PPAR $\gamma 2$ promoter (Dani 1999) and reinforces the expression of preadipocyte factor (PREF-1) (Lian et al. 2004); (iii) PREF-1, is synthetized as a protein for the plasmatic membrane and it maintains the preadipocyte phenotype thus inhibiting adipogenesis. Dexamethasone, one of the adipogenic inductors inhibits the transcription of PREF-1 (Smas et al. 1997).

\section{Signaling pathways involved in adipoge- nesis}

In order for the process of adipogenic differentiation to take place there are certain signaling pathways that must be activated or silenced and two of them are as follows:

\section{a. Wnt signaling pathway}

Many researchers have experimented on 3T3-L1 and 3T3-F442A cells to find out the role of Wnt signaling pathway in adipogenesis and they arrived to the conclusion that this pathway inhibits the process by interfering with the cell cycle and blocking the expression of PPAR- $\gamma$ and C/EBP- $\alpha$ (Ross et al. 2000; Bennett et al. 2002). Wingless type MMTV integration site family member 10b (Wnt10b), an activator for the signaling pathway is strongly expressed in preadipocytes, but its level of expression reduces dramatically with the initiation of adipogenic differentiation (Moldes et al. 2003). The Wnt signaling mechanism is presented in Figure 3.

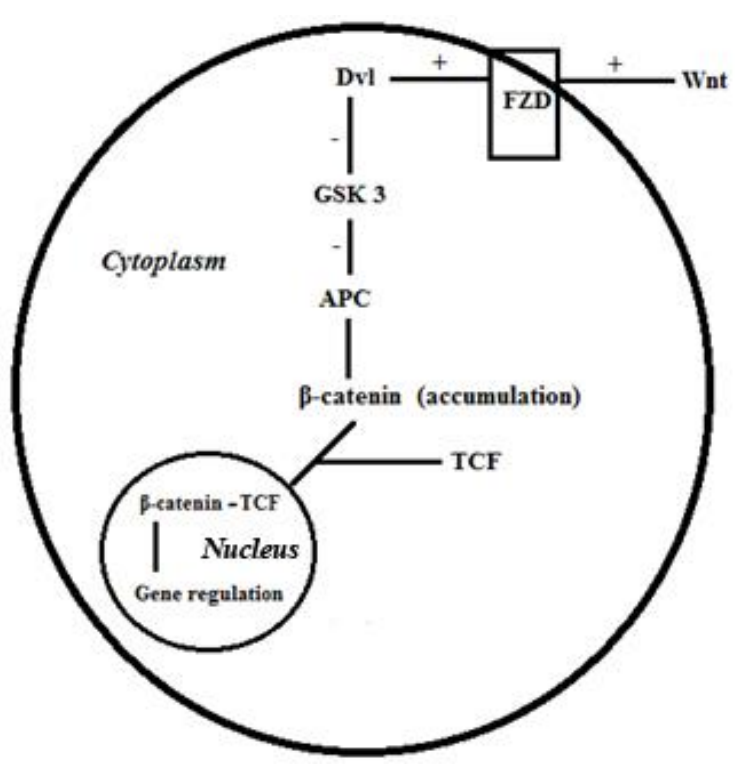

Fig. 3. Wnt signaling pathway. Wnt glycoproteins bind to the transmembrane receptor Frizzled (FZD). This determines the translocation of axin into the plasmatic membrane and the phosphorylation of Disheveled protein (Dvl). The activated Dvl inhibits glycogen synthase kinase 3, which in turn inhibits adenomatosis polyposis coli (APC) protein. These events prevent the degradation of $\beta$-catenin, which instead accumulates in the cytoplasm of the cell. Afterwards it is translocated to the nucleus, where it acts as a co-activator for the transcription factors LEF/TCF, activating the genes targeted by Wnt.

\section{b. Hedgehog signaling pathway}

This signaling pathway is an important modulator of the differentiation processes, including adipogenesis. It has been demonstrated that activation of the Hedgehog signaling pathway inhibits adipogenic differentiation and 
promotes osteogenesis in 3T3-L1, NIH-3T3 and mesenchymal cells by reducing the expression of adipogenic markers ( $\mathrm{C} / \mathrm{EBP} \alpha, \operatorname{PPAR} \gamma, \mathrm{AP} 2$, adipsin), enhancing the expression of anti-adipogenic transcription factors (PREF-1, GATA2) and inducing the expression of osteogenic markers: RUNX2, alkaline phosphatase (ALP) and osteomodulin (Wu et al. 2004; Suh et al. 2006; Fontaine et al. 2008).

Hedgehog signaling proteins attach themselves to the complex of Patched receptors (Ptc1 and Ptc2), which have a negative effect over Hedgehog proteins and the Smoothened transmembrane receptor (Smo) located on the surface of the cells. The generated signal is transmitted to the nucleus and gene expression is controlled through the family of transcription factors ci/Gli. In the first stages of adipogenesis expression of the genes which encode for Smo and Gli is reduced, while the expression of the genes responsible for the Ptc1 and Ptc2 receptors increases (McMahon et al. 2003).

\section{Conclusions}

Adipose tissue engineering represents a multidisciplinary field with extraordinary potential for the regeneration of adipose tissue. The main strategy involves the use of cells (that can differentiate into adipocytes), biomaterials also called scaffolds (which act as a support for the cells seeded on their surface) and induction factors that mediate the process of adipogenic differentiation. The resulting bioconstruct has to be compatible with the host, bioactive and resistant for a long period of time in order for it to act as a substitute and be implanted at the targeted site of regeneration.

Adipogenesis is a multiple stage process, which involves the expression of specific genes and the silencing of others, unimportant for the development of precursor cells into mature adipocytes. The most important transcription factors are PPAR $\gamma$ and $\mathrm{C} / \mathrm{EBP} \alpha$, and they act in a cooperatively manner to regulate the expression of specific adipose genes. Adipogenic differentiation is mediated by certain signaling pathways, two of them being Wnt and Hedgehog signaling pathways.

The long-term perspectives of the studies performed on adipogenesis involve reducing the rate of obesity and diabetes. Furthermore, adequate ATE strategies can be developed by knowing in detail the process of adipogenesis. There is still a lot to explore but in the future, therapies based on ATE products might be able to replace current clinical procedures, which have numerous limitations.

\section{Acknowledgments}

The Authors acknowledge their formation in the field of tissue engineering and cell differentiation by 65PCCDI project and PCCE 248/2010 national grant. No funding has been received for the preparation of the manuscript.
(C)The Author(s) 2018

Open Access This article is distributed under the terms of the Creative Commons Attribution 4.0 International License (http://creativecommons.org/licenses/by/4.0/) which permits unrestricted use, distribution, and reproduction in any medium, provided you give appropriate credit to the original author(s) and the source, provide a link to the Creative Commons license, and indicate if changes were made.

\section{References}

Ailhaud G. 1996. Early adipocyte differentiation. Biochem. Soc. Trans. 24, 400-402.

Altiok S., Xu M., Spiegelman B.M. 1997. PPAR gamma induces cell cycle withdrawal: inhibition of E2F/DP DNA-binding activity via down-regulation of PP2A. Genes Dev. 11, 1987-1998.

Amri E.Z., Bonino F., Ailhaud G., Abumrad N.A., Grimaldi P.A. 1995. Cloning of a protein that mediates transcriptional effects of fatty acids in preadipocytes. Homology to peroxisome proliferatoractivated receptors. J. Biol. Chem. 270, 2367-2371.

Bennett C., Ross S., Longo K., Bajnok L., Hemati N., Johnson K., Harrison S., MacDougald O. 2002. Regulation of Wnt signaling during adipogenesis. $J$. Biol. Chem. 277, 30998-31004.

Braissant O., Foufelle F., Scotto C., Dauca M., Wahli W. 1996. Differential expression of peroxisome proliferator-activated receptors (PPARs): tissue distribution of PPAR in the adult rat. Endocrinology. 137, 354-366.

Brand-Saberi B. 2005. Genetic and epigenetic control of skeletal muscle development. Ann. Anat. 187, 199207.

Cannon B., Nedergaard J. 2004. Brown adipose tissue: function and physiological significance. Physiol. Rev. 84, 277-359.

Casadei A., Epis R., Ferroni L., Tocco I., Gardin C., Bressan E., Sivolella S., Vindigni V., Pinton P., Mucci G., Zavan B. 2012. Adipose tissue regeneration: a state of the art. J. Biomed. Biotech. 2012, 1-12.

Chargé S.B., Rudnicki M.A. 2004. Cellular and molecular regulation of muscle regeneration. Physiol. Rev. 84, 209-238.

Choi J., Gimble J., Lee K., Marra K., Rubin J., Yoo J., Vunjak-Novakovic G., Kaplan D. 2010. Adipose tissue engineering for soft tissue regeneration. Tissue Eng. 16, 413-426.

Christy R., Kaestner K., Geiman D., Lane M. 1991. CCAAT/enhancer binding protein gene promoter: binding of nuclear factors during differentiation of 3T3-L1 preadipocytes. Proc. Natl. Acad. Sci. USA. 88, 2593-2597.

Coleman S. 1995. Long-term survival of fat transplants: controlled demonstrations. Aesthet. Plast. Surg. 19, 421-425.

Dani C. 1999. Embryonic stem cell-derived adipogenesis. Cells Tissues Organs. 165, 173-180. 
Dicker A., Le Blanc K., Astrom G., van Harmelen V., Götherström C., Blomqvist L., Arner P., Rydén M. 2005. Functional studies of mesenchymal stem cells derived from adult human adipose tissue. Exp. Cell. Res. 308, 283-290.

Dinescu S. 2015. Cellular and molecular mechanisms of adipose-derived stem cells differentiation. PhD thesis, University of Bucharest, Bucharest, Romania.

Fajas L., Auboeuf D., Raspe E., Schoonjans K., Lefebvre A-M. 1997. The organization, promoter analysis, and expression of the human PPAR gene. $J$. Biol. Chem. 272, 18779-18789.

Farmer S. 2008. Molecular determinants of brown adipocyte formation and function. Genes Dev. 22, 1269-1275.

Fischbach C., Seufert J., Staiger H., Hacker M., Neubauer M., Göpferich A., Blunk T. 2004. Threedimensional in vitro model of adipogenesis: comparison of culture conditions. Tissue Eng. 10, 215-229.

Flynn L., Semple J., Woodhouse K. 2006. Decellularized placental matrices for adipose tissue engineering. J. Biomed. Mater. Res. Part A. 79A, 359-369.

Flynn L., Woodhouse K. 2008. Adipose tissue engineering with cells in engineered matrices. Organogenesis. 4, 228-235.

Fontaine C., Cousin W., Plaisant M., Dani C., Peraldi P. 2008. Hedgehog signaling alters adipocyte maturation of human mesenchymal stem cells. Stem Cells. 26, 1037-1046.

Franchini M. 2003. Mesenchymal stem cells: from biology to clinical applications. Recenti. Prog. Med. 94, 478-483.

Fuchs J., Nasseri B., Vacanti J. 2001. Tissue engineering: a 21 st century solution to surgical reconstruction. Ann. Thorac. Surg. 72, 577-591.

Gesta S., Tseng Y., Kahn C. 2007. Developmental origin of fat: tracking obesity to its source. Cell. 131, 242-256.

Gomillion C., Burg K. 2006. Stem cells and adipose tissue engineering. Biomaterials. 27, 6052-6063.

Greenberg A.S., Egan J.J., Wek S.A., Moos M., Londos C., Kimmel A.R. 1993. Isolation of cDNAs for perilipins A and B: sequence and expression of lipid droplet-associated proteins of adipocytes. Proc. Natl. Acad. Sci. USA. 90, 12035-12039.

Griffith L., Naughton G. 2002. Tissue engineering-current challenges and expanding opportunities. Science. 295, 1009-1014.

Hausman D., DiGirolamo M., Bartness T., Hausman G., Martin R. 2001. The biology of white adipocyte proliferation. Obes. Rev. 2, 239-254.

Howard D., Buttery L., Shakesheff K., Roberts S. 2008. Tissue engineering: strategies, stem cells and scaffolds. J. Anat. 213, 66-72.

Huss F., Kratz G. 2002. Adipose tissue processed for lipoinjection shows increased cellular survival in vitro when tissue engineering principles are applied. Scand. J. Plast. Reconstr. Surg. Hand Surg. 36, 166-171.

Hwang C., Mandrup S., MacDougald O., Geiman D., Lane M. 1996. Transcriptional activation of the mouse obese $(\mathrm{Ob})$ gene by CCAAT/enhancer binding protein alpha. Proc. Natl. Acad. Sci. USA. 93, 873-877.

Ibrahimi A., Sfeir Z., Magharaie H., Amri E. Z., Grimaldi P., Abumrad N. A. 1996. Expression of the CD36 homolog (FAT) in fibroblast cells: effects on fatty acid transport. Proc. Natl. Acad. Sci. USA. 93, 2646-2651.

Kang S., Seo S., Choi C., Kim B. 2008. Porous poly(lactic-co-glycolic acid) microsphere as cell culture substrate and cell transplantation vehicle for adipose tissue engineering. Tissue Eng. 14, 25-34.

Katz A., Llull R., Hedrick M., Futrell J. 1999. Emerging approaches to the tissue engineering of fat. Clin. Plast. Surg. 26, 587-603.

Kirkland J., Hollenberg C., Kindler S., Gillon W. 1994. Effects of age and anatomic site on preadipocyte number in rat fat depots. J. Gerontol. 49, B31-35.

Lavik E., Langer R. 2004. Tissue engineering: current state and perspectives. Appl. Microbiol. Biotechnol. $65,1-8$.

Lefterova M., Lazar M. 2009. New developments in adipogenesis. Trends Endocrinol. Metab. 20, 107114.

Levenberg S., Langer R. 2004. Advances in tissue engineering. Curr. Top. Dev. Biol. 61, 113-134.

Lian J.B., Javed A., Zaidi S.K. 2004. Regulatory controls for osteoblast growth and differentiation: role of Runx/Cbfa/AML factors. Crit. Rev. Eukaryot. Gene Expr. 14, 1-41.

Lin F., Lane M. 1994. CCAAT/enhancer binding protein alpha is sufficient to initiate the 3T3-L1 adipocyte differentiation program. Proc. Natl. Acad. Sci. USA. 91, 8757-8761.

Lin S., Wang K., Kao A. 2008. Engineered adipose tissue of predefined shape and dimensions from human adipose-derived mesenchymal stem cells. Tissue Eng. 14, 571-581.

Locke M., de Chalain T. 2008. Current practice in autologous fat transplantation. Ann. Plast. Surg. 60, 98-102.

MacDougald O.A., Hwang C.S., Fan H., Lane M.D. 1995. Regulated expression of the obese gene product (leptin) in white adipose tissue and 3T3-L1 adipocytes. Proc. Natl. Acad. Sci. USA. 92, 90349037.

MacDougald O.A., Lane M.D. 1995. Transcriptional regulation of gene expression during adipocyte differentiation. Annu.. Rev. Biochem. 64, 345-373.

Mandrup S., Lane M.D. 1997. Regulating adipogenesis. J. Biol. Chem. 272, 5367-5370.

Marra K., DeFail A., Clavijo-Alvarez J., Badylak S., Taieb A., Schipper B., Bennett J., Rubin J. 2008. FGF-2 enhances vascularization for adipose tissue engineering. Plast. Reconstr. Surg. 121, 1153-1164. 
McMahon A., Ingham P., Tabin C. 2003. Developmental roles and clinical significance of hedgehog signaling. Curr. Top. Dev. Biol. 53, 1-114.

Moldes M., Zuo Y., Morrison R., Silva D., Park B., Liu J., Farmer S. 2003. Peroxisome-proliferator-activated receptor $\mathrm{G}$ suppresses $\mathrm{Wnt} / \mathrm{b}-\mathrm{Catenin}$ signaling during adipogenesis. Biochem J. 376, 607-613.

Monfort A., Izeta A. 2012. Strategies for human adipose tissue repair and regeneration. JCDSA. 2, 93-107.

Moreno-Navarrete J., Fernández-Real J. 2012. Adipocyte differentiation. In: Adipose Tissue Biology, Symonds M.E. (Ed.), New York, Springer, 17-39.

Moseti D., Regassa A., Kim W. 2016. Molecular regulation of adipogenesis and potential antiadipogenic bioactive molecules. Int. J. Molec. Sci. 17, $1-24$.

Moustaid N., Sul H.S. 1991. Regulation of expression of the fatty acid synthase gene in 3T3-L1 cells by differentiation and triiodothyronine. J. Biol. Chem. 266, 18550-18554.

Nguyen A., Pasyk K., Bouvier T., Hassett C., Argenta L. 1990. comparative study of survival of autologous adipose tissue taken and transplanted by different techniques. Plast. Reconstr. Surg. 85, 378-389.

Otto W.R., Rao J. 2004. Tomorrow's skeleton staff: mesenchymal stem cells and the repair of bone and cartilage. Cell Prolif. 37, 97-110.

Pairault J., Green H. 1979. A study of the adipose conversion of suspended 3T3 cells by using glycerophosphate dehydrogenase as differentiation marker. Proc. Natl. Acad. Sci. USA. 76, 5138- 5142.

Patrick C. 2001. Tissue engineering strategies for adipose tissue repair. Anat. Rec. 263, 361-366.

Patrick C., Chauvin P., Hobley J., Reece G. 1999. Preadipocyte seeded PLGA scaffolds for adipose tissue engineering. Tissue Eng. 5, 139-151.

Patrick C., Zheng B., Johnston C., Reece G. 2002. Long-term implantation of preadipocyte-seeded PLGA scaffolds. Tissue Eng. 8, 283-293.

Ramji D., Foka. P. 2002. CCAAT/enhancer-binding proteins: structure, function and regulation. Biochem J. 365, 561-575.

Ross S., Hemati N., Longo K., Bennett C., Lucas P., Erickson R., MacDougald O. 2000. Inhibition of adipogenesis by Wnt signaling. Science. 289, 950953.

Rustad K., Sorkin M., Levi B., Longaker M., Gurtner G. 2010. Strategies for organ level tissue engineering. Organogenesis. 6, 151-157.

Schipper B., Marra K., Zhang W., Donnenberg A., Rubin J. 2008. Regional anatomic and age effects on cell function of human adipose-derived stem cells. Ann. Plast. Surg. 60, 538-544.

Schoonjans K., Staels B., Auwerx J. 1996. The peroxisome proliferator activated receptors (PPARS) and their effects on metabolism and adipocyte differentiation. Biochim. Biophys. Acta. 1302, 93-109.
Scott M., Nguyen V., Levi B., James A. 2011. Current methods of adipogenic differentiation of mesenchymal stem cells. Stem Cells Dev. 20, 17931804.

Shanti R., Janjanin S., Li W., Nesti L., Mueller M., Tzeng M., Tuan R. 2008. In vitro adipose tissue engineering using an electrospun nanofibrous scaffold. Ann. Plast. Surg. 61, 566-571.

Smas C.M., Chen L., Sul H.S. 1997. Cleavage of membrane- associated PREF-1 generates a soluble inhibitor of adipocyte differentiation. Mol. Cell. Biol. 17, 977-988.

Sommer B., Sattler G. 2000. Current concepts of fat graft survival: histology of aspirated adipose tissue and review of the literature. Dermatol. Surg. 26, 1159-1166.

Spiegelman B.M., Frank M., Green H. 1983. Molecular cloning of mRNA from $3 \mathrm{~T} 3$ adipocytes. Regulation of mRNA content for glycerophosphate dehydrogenase and other differentiation-dependent proteins during adipocyte development. J. Biol. Chem. 258, 1008310089.

Spiegelman B.M., Choy L., Hotamisligil G.S., Graves R.A., Tontonoz P. 1993. Regulation of adipocyte gene expression in differentiation and syndromes of obesity/diabetes. J. Biol. Chem. 268, 6823-6826.

Sugihara H., Yonemitsu N., Miyabara S., Yun K. 1986. Primary cultures of unilocular fat cells: characteristics of growth in vitro and changes in differentiation properties. Differentiation. 31, 42-49.

Suh J., Gao X., McKay J., McKay R., Salo Z., Graff J. 2006. Hedgehog signaling plays a conserved role in inhibiting fat formation. Cell Metab. 3, 25-34.

Tabata Y., Miyao M., Inamoto T., Ishii T., Hirano Y., Yamaoki Y., Ikada Y. 2000. De novo formation of adipose tissue by controlled release of basic fibroblast growth factor. Tissue Eng. 6, 279-289.

Tajbakhsh S. 2005. Skeletal muscle stem and progenitor cells: Reconciling genetics and lineage. Exp. Cell Res. 306, 364-372.

Tilg H., Moschen A. 2006. Adipocytokines: mediators linking adipose tissue, inflammation and immunity. Nat. Rev. Immunol. 6, 772-783.

Tontonoz P., Hu E., Graves R. A., Budavari A. I., Spiegelman B. M. 1994. mPPAR gamma 2: tissuespecific regulator of an adipocyte enhancer. Genes Dev. 8, 1224-1234.

Tontonoz P., Hu E., Devine J., Beale E.G., Spiegelman B. M. 1995. PPAR gamma 2 regulates adipose expression of the phosphoenolpyruvate carboxykinase gene. Mol. Cell. Biol. 15, 351-357.

Torti F.M., Torti S.V., Larrick J.W., Ringold G.M. 1989. Modulation of adipocyte differentiation by tumor necrosis factor and transforming growth factor beta. J. Cell Biol. 108, 1105-1113.

Umek R.M., Friedman A.D., McKnight S.L. 1991. CCAAT/enhancer binding protein: a component of a differentiation switch. Science. 251, 88-292. 
Vermette M., Trottier V., Menard V., Saint-Pierre L., Roy A., Fradette J. 2007. Production of a new tissueengineered adipose substitute from human adiposederived stromal cells. Biomaterials. 28, 2850-2860.

Wilkison W.O., Min H.Y., Claffey K.P., Satterberg B.L., Spiegelman B.M. 1990. Control of the adipsin gene in adipocyte differentiation. Identification of distinct nuclear factors binding to single and doublestranded DNA. J. Biol. Chem. 265, 477-482.

Wu X., Walker J., Zhang J., Ding S., Schultz P.G. 2004. Purmorphamine induces osteogenesis by activation of the hedgehog signaling pathway. Chem. Biol. 11, $1229-1238$.

Zuk P.A., Zhu M., Mizuno H., Huang J., Futrell J.W., Katz A.J., Benhaim P, Lorenz H.P., Hedrick M.H. 2001. Multilineage cells from human adipose tissue: implications for cell-based therapies. Tissue Eng. 7, 211-228.

Zuo Y., Quiang L., Farmer S. 2006. Activation of CCSST/enhancer-binding protein (C/EBP) $\alpha$ expression by $\mathrm{C} / \mathrm{EBP} \beta$ during adipogenesis requires a peroxisome proliferator-activated receptor- $\gamma$ associated repression of HDAC1 and $\mathrm{C} / \mathrm{EBP} \alpha$ gene promoter. J. Biol. Chem. 281, 7960-7967. 